\title{
SÉANCE DU 27 JUIN 1919
}

Présidence du Prince bonaparte.

M. Moreau donne lecture du procès-verbal de la dernièré séance, dont la rédaction est adoptée.

M. le Président a le regret d'annoncer le décès de notre confrère M. J. Joigny.

M. le Président annonce ensuite les présentations suivantes :

MM. Emberger, pharmacien, licencié ès sciences, au laboratoire de Botanique de la Faculté des Sciences de Lyon, par MM. Guilliermond et Moreau.

Beauverie (Jean), professeur adjoint à la Faculté des Sciences de Nancy, par MM. Guilliermond et Magnin.

Bertravd (Paul), maître de conférences à la Faculté des Sciences de Lille, rue Brut-Maison, $n^{\circ} 159$, à Lille, par MM. le Prince Bonaparte et Lutz.

Maison Vilmorin-AndrieuX et $\mathrm{C}^{\text {le }}$, à Verrières-leBuisson (Seine-et-0ise), par MM. Jacques de Vilmorin et le Prince Bonaparte.

Simox (Eugène), receveur de l'enregistrement à Montmorillon (Vienne), par MM. Giraudias et Coste.

Suivant l'usage momentanément en vigueur, MM. Emberger, Beauverie, Bertrand, la Maison Vilmorin-Andrieux Lxvı. (SÉANCES) 15 
et $C^{\text {lee }}$, M. Simon sont proclamés membres de la Société.

Suivant avis de M. le Trésorier, M. Bertrand, ayant rempli les conditions prescrites par les statuts, est proclamé membre à vie.

Le Prince Bonaparte fait hommage à la Société des fascicules $\mathrm{V}$ et VII de ses Notes Ptéridologiques qui renferment les déterminations d'environ 2500 spécimens de Fougères provenant de toutes les parties du globe. 29 espèces et 19 variétés nouvelles y sont rituellement décrites. Le fascicule VII contient le début d'une Flore ptéridologique descriptive et analytique complète de l'Indo-Chine dont la suite, actuellement à l'impression, paraîtra dans les pròchains fascicules.

Le Prince Bonaparte annonce en même temps qu'à sa demande, M. Carl Christensen, de Copenhague, a publié un nouveau supplément à son Index Filicum qui, c९mme on le sait, a déjà rendu tant de services aux ptéridologues en unifiant la nomenclature des Fougères parfois si embrouillée. Dès que les moyens, de transport le permettront, cette publication lui sera envoyée de Copenhague, et il se fera un plaisir d'en offrir un exemplaire à la Société ainsi qu'à tous ceux de ses collègues qui voudront bien le lui demander.

\section{DONS FAITS A LA SOCIÉTÉ}

Bernard (Noël), L'évolution des plantes.

Burnat (Emile), Flore des Alpes-Maritimes, VI, 2, par John Briquet et François Cavillier.

Candolle (C. et A.), Sur la ramification des Sequoia.

Cockayne (A.-H.), Some economic considerations concerning Montana Tussock Grassland.

Cockayne and Foweraker (C.-A.), The Principal Plant Associations of the immediate Vicinity of the Station (Canterbury Mountain Biological Station, New Zealand).

Douin(Ch.), Les variations du Gamétophytechez les Céphaloziellacées. 
Fragoso (R.-G.), Bosquejo de una Florula hispalense de Micromicetos.

Gadeau de Kerville (Henri), Notes sur les Fougères, 1-รั.

- Considérations et recherches expérimentales sur la direction des racines et des tiges.

Gerbault (Éd.), Note sur le Sedum micranthum.

- Viola eburnea.

- Absence héréditaire de l'éperon floral dans une lignée de Linaria Cymbalaria Mill.

Grunow Alberto (1826-1914) [Notizia biographiche di Achille Forti]. Lecomte (H.), Notulæ Systematicx, III, 9-10.

Letacq et Gerbault, Note sur la flore de Mézangers (Mayenne).

Maire (R.), Champignons Nord-Africains nouveaux ou peu connus.

- Maladies des végétaux ligneux de l'A friqué du Nord.

- La flore mycologique des forêts de Cèdres de l'Atlas.

- Annotalions à la flore de l'Algérie, $\mathrm{I}$. Tunisie.

- Deux Contributions à l'étude de la flore mycologique de la

- Remarques sur le Protascus subuliformis.

- Contribution à l'élude de la flore forestière du Moyen Atlas marocain.

- Schedic ad Mycothecam Boreali-Africanam, $\mathrm{n}^{\text {os }}$ 1-2כ0.

- Sur une nouvelle Laboulbéniale parasite des Scaphidiidx.

- Deuxième Contribution à l'étude des Laboulbéniales de l'Afrique du Nord.

- Deuxième Contribution à l'étude de la flore du Djurjura.

- Sur quelques Laboulbeniales.

- Les Champignons vénéneux de l'Algérie.

- La végétation des montagnes du Sud-Oranais.

Mameli (E.) e Cattaneo (E.), Sul geotropismo negativo spontaneo di radici di Helianthus annuus e di alcune altre piante.

Montemartini (Luigi), Intorno ad alcuni casi di simbiosi autumnale locale temporane.

Toni (G.-B. de), La Nuova Notarisia, XXVIII.

- Rassegna di monstruosita florali in individuei di Digitalis purpurea $L$.

- Nuove osservazioni di teratologia forale mella Digitalis purpurea $L$.

Toni (G.-B. de) e Forti (Achille), Catalogo delle Alghe raccolte nella regione di Bengasi dal R.P. D. Vito Zanon. 
- Seconda... e Terza contribuzione alla flora algologica della Libia italiana.

- Analisi microscopica di alcuni saggi di fitoplancton raccolti della R. N. Liguria.

Tromp (R.-D.), The work of the Forest Department in India.

Turconi (M.), Sopra una nova malattia del Cacao.

Mémoires de la Socièté d'Agriculture, Sciences et Arts d'Angers, $\mathrm{S}^{\mathrm{e}}$ série, XIX, 1916; XX, 1917.

Bulletin de la Société scientifique de l'Aude, XXV, 1814.

Bulletin de la Société française pour l'échange des plantes, $6^{\mathrm{e}}$ fasc., 1916.

Nouveaux Mémoires de la Société helvétique des Sciences naturelles, LII.

The Indian Forest Record, VII, 1-3.

Forest Bulletin, $\mathrm{n}^{\text {os }} 33,33,36,37$.

Memoirs of the Department of Agriculture in India, IX.

Proceedings of the National Academy of Science of the United States of America, III.

Transactions of the R. Society of Canada, Ser. 3, X.

Journal of Agricultural Research, X, 3.

Il est donné connaissance des communications suivantes :

\section{Sertum plantarum novarum. Pars secunda;}

Par M. Michei. GaNDOYER.

Cyenium dentiflorum Gdgr. - Aphyllum, villoso-glandulosum, caulis flexuosus uniflorus, folia caulinia (seu bracteæ) squamiformia, ovato-obtusa, tubus ovatus hirtus, vix nervosus, ejus dentes triangulares acuti, tubo villoso saltem duplo breviores, corollæ ad faucem glabræ magnæ, 4-lobatæ lobis glabris orbiculato obtusis, basi attenuatis, valde serratis vel incisis.

HAB. : Cap, ad Port Elisabeth (Laidley E. S. C. A. n. 212!).

Cum C. tuhato Benth. tantum comparari potest a quo valde differt indumento glanduloso, tubæ corollæ incisæ 4-5-plo breviore, etc.

Cycnium rectum Gdgr. - Scabroso-pubescens, foliosum, rectum, caulis adpresse hirtus, folia angustius oblongo-acuta, basi longe attenuata, petiolata, scaberrima, remote parceque serrata, calyx breviter hirtus, tubo villosulo duplo brevior, sepala cuspidata, corollæ lóbi integri, plani, suborbiculati, faux glabra. 


\section{$2 \mathrm{BHL}$ Biodiversity Heritage Library}

1919. "Séance Du 27 Juin 1919." Bulletin de la Société botanique de France 66, 213-216. https://doi.org/10.1080/00378941.1919.10836100.

View This Item Online: https://www.biodiversitylibrary.org/item/8686

DOI: https://doi.org/10.1080/00378941.1919.10836100

Permalink: https://www.biodiversitylibrary.org/partpdf/161139

\section{Holding Institution}

Missouri Botanical Garden, Peter H. Raven Library

\section{Sponsored by}

Missouri Botanical Garden

\section{Copyright \& Reuse}

Copyright Status: Public domain. The BHL considers that this work is no longer under copyright protection.

This document was created from content at the Biodiversity Heritage Library, the world's largest open access digital library for biodiversity literature and archives. Visit BHL at https://www.biodiversitylibrary.org. 